\title{
Separate CT-Reconstruction for 3D Wavelet Based Noise Reduction Using Correlation Analysis
}

\author{
Anja Borsdorf ${ }^{1}$, Rainer Raupach ${ }^{2}$, and Joachim Hornegger ${ }^{1}$
}

\begin{abstract}
The projection data measured in computed tomography (CT) and, consequently, the volumes reconstructed from these data contain noise. For a reliable diagnosis and subsequent image processing, like segmentation, the ratio between relevant tissue contrasts and the noise amplitude must be sufficiently large. We propose a novel 3D wavelet based method for structurepreserving noise reduction in CT. By separate reconstructions from disjoint subsets of projections, two volumes can be computed, which only differ with respect to noise. Two disjoint subsets of projections can be directly acquired using a dualsource CT-scanner. Otherwise, the two volumes can be generated by reconstructing even and odd numbered projections separately. Correlation analysis between the approximation coefficients of the two input datasets, combined with an orientation and position dependent noise estimation are used for differentiating between structure and noise at each level of the wavelet decomposition. The proposed method adapts itself to the locally varying noise power and allows an anisotropic denoising. The quantitative and qualitative evaluation on phantom and clinical data showed that noise reduction rates up to $60 \%$ can be achieved without noticeable loss of resolution.
\end{abstract}

Index Terms-Computed Tomography, Noise Reduction, Wavelets, Correlation Analysis

\section{INTRODUCTION}

$\mathbf{I}$ $\mathrm{N} \mathrm{CT}$, the projections acquired at the detector and, consequently, the volumes reconstructed from these data contain quantum noise. This noise can, for example, be reduced by increasing the radiation dose or by choosing a smoothing reconstruction [1]. However, with respect to patients' care, the least possible radiation dose is required and a smoothing reconstruction lowers image resolution. This shows that pixel noise cannot be reduced arbitrarily. Nevertheless, an increased signal-to-noise ratio is beneficial for a reliable diagnosis and subsequent image processing. Many different approaches for noise suppression in CT have been investigated. There are, for example, iterative numerical reconstruction techniques based on optimizing statistical objective functions [2]-[4]. Furthermore, several linear or nonlinear filtering methods for noise reduction in the sinogram [5]-[7] have been proposed. Structure-preserving denoising in sinogram-space is difficult because of the low signal-to-noise ratio in the projections. Therefore, in this work a new noise suppression method

${ }^{1}$ A. Borsdorf and J. Hornegger are with with the FriedrichAlexanderUniversity ErlangenNuremberg (FAU), Institute of Pattern Recognition, Martensstr. 3, 91058 Erlangen, Germany (see http://www5.informatik.unierlangen.de).

${ }^{2}$ R. Raupach is with Siemens Medical Solutions, Siemensstr. 1, 91301 Forchheim, Germany.

The concepts and information presented in this paper is based on research and is not commercially available. working on the reconstructed data is proposed. Reducing noise after CT-reconstruction is challenging mainly due to the difficult noise properties: After reconstruction, the noisedistribution is not known. Noise is non-stationary and directed noise due to strong attenuation along certain directions might be present. This is the reason why many common noise suppression methods fail or lead to unconvincing results on CT data. We present a 3D wavelet based denoising method that adapts itself to the noise power and allows for the reduction of spatially varying and oriented noise without noticeable loss of resolution.

\section{PREVIOUS WORK}

Recently, Tischenko et al. [8] proposed a structure-saving noise reduction method using the correlations between two images for threshold determination in the wavelet domain. They used approximated gradients from the detail coefficients of an á-trous wavelet transformation for edge-detection, in order to denoise two projection radiography images taken shortly one after another. In [9] we proposed a more general method and described how separate reconstructions from even and odd projections respectively can be used for denoising CT slices based on correlation analysis in the wavelet domain. Although we achieved a respectable noise reduction there were two problems remaining: The larger the local neighborhood used for correlation analysis at a certain position the more reliable was the computation. However, lower noise reduction was achieved around edges with higher contrast. The second limitation was that all the detail coefficients, i.e. all orientations, at one decomposition level were weighted equally, not allowing the noise reduction along an edge or stronger directed noise. In [10] we showed how a local and orientation dependent noise estimation from the differences between the detail coefficients of the two separately reconstructed images can be used for noise-adaptive thresholding in the wavelet domain.

\section{METHOD}

We now present a method that combines the advantages of both our previous approaches. A correlation analysis between the approximation coefficients of the two input datasets, combined with an orientation and position dependent noise estimation is used for differentiating between structure and noise. Furthermore, we investigate the extension of the method to $3 \mathrm{D}$, which additionally leads to a more reliable correlation analysis and fewer side effects regarding the preservation of edges. 


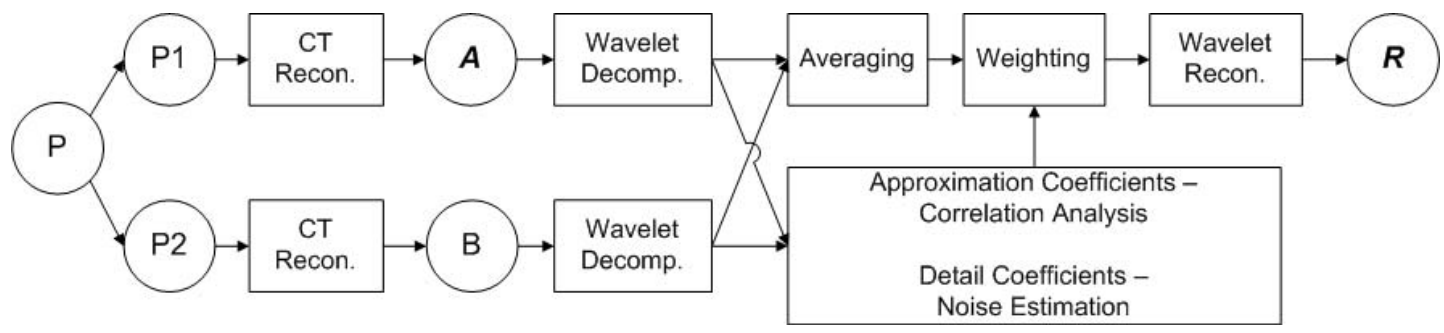

Fig. 1. Block diagram of the noise reduction method.

\section{A. Overview}

An overview of the methodology can be found in Fig. 1 . First two volumes $A$ and $B$ are reconstructed from disjoint subsets of projections $\mathrm{P} 1 \subset \mathrm{P}$ and $\mathrm{P} 2 \subset \mathrm{P}$, with $\mathrm{P} 1 \cap \mathrm{P} 2=\emptyset$, $|\mathrm{P} 1|=|\mathrm{P} 2|$ and $\mathrm{P}=\mathrm{P} 1 \cup \mathrm{P} 2$, where $|\mathrm{P}|$ defines the number of samples in $\mathrm{P}$. The projections $\mathrm{P} 1$ and $\mathrm{P} 2$ can for example directly be acquired using a dual-source CTscanner. Alternatively, one image can be reconstructed from the even and the other from the odd numbered projections of a single scan. In both cases, noise between the projections can be assumed to be uncorrelated (see [11], [12] for more information about $\mathrm{CT}$ reconstruction and the propagation of noise from the projections to the reconstructed slices). In the following, we assume that the sampling theorem is fulfilled for both single sets of projections. Consequently, $A$ and $B$ only differ with respect to image noise, but include the same ideal noise-free signal:

$$
A=S+N_{A}, \quad B=S+N_{B},
$$

where $S=\mathrm{E}(A)=\mathrm{E}(B)$ represents the ideal noise-free data (the statistical expectation E) and $N_{A} \neq N_{B}$ zero-mean noise $\left(\mathrm{E}\left(N_{A}\right)=\mathrm{E}\left(N_{B}\right)=0\right)$ included in $A$ and $B$, respectively. Noise in both datasets is non-stationary, and consequently the standard deviation of noise depends on the local position $\mathbf{x}=\left(x_{1}, x_{2}\right)$ in $2 \mathrm{D}$ or $\mathbf{x}=\left(x_{1}, x_{2}, x_{3}\right)$ in $3 \mathrm{D}$. The standard deviations at a given position are approximately the same:

$$
\sigma_{A}(\mathbf{x}) \approx \sigma_{B}(\mathbf{x}),
$$

because on average the same number of contributing quanta can be assumed. Noise between the projections P1 and P2 is uncorrelated and accordingly noise between the separately reconstructed images is uncorrelated, too, leading to the following covariance:

$$
\operatorname{Cov}\left(N_{A}, N_{B}\right)=0 .
$$

These properties are considered during the denoising process, which can be applied either to the $2 \mathrm{D}$ slices or the $3 \mathrm{D}$ volumes. Both datasets are decomposed by a $2 \mathrm{D}$ or 3D discrete dyadic wavelet transformation. After this linear transformation, for example of the input dataset $A=A_{0}$, four two-dimensional or eight three-dimensional blocks of coefficients are available at each decomposition level $l$ : the lowpass filtered approximation $A_{l}$ and the highpass filtered details $W_{l}^{\mathrm{d}}$, where d describes the direction in space. For the 2D case, e.g., d can be the horizontal, vertical or diagonal direction. The detail coefficients include high frequency structures together with noise in the respective frequency bands and orientations. In the following the wavelet representations of the two input datasets are used for differentiating between detail coefficients that belong to structure and noise, and to compute weighting coefficients accordingly. These weights consist of two parts: a correlation coefficient based weight and a significance-weight, which are both described more in detail in the following section. The computed weights are then applied to the averaged wavelet coefficients of the input datasets, ensuring that the entire acquired information is used for the final result $R$.

\section{B. Correlation Analysis}

At each decomposition level, a local correlation analysis between the approximation coefficients of $A$ and $B$ is performed. This leads to one block of correlation coefficients having the same size as the detail coefficients at the respective decomposition level.

A very close connection between the detail coefficients and the correlation analysis can be obtained if the approximation coefficients of the previous decomposition level $l-1$ are used for correlation analysis at level $l$. The detail coefficients at level $l$ are computed from the approximation coefficients at level $l-1$ and these values are also used for correlation analysis at the respective position. For the correlation based weight $G_{l}\left(\mathbf{x}_{l}\right)$, the empirical correlation coefficient [13] is computed. Approximation coefficients $A_{l-1}$ and $B_{l-1}$ within a local neighborhood $\Omega_{\mathrm{x}}$ around the corresponding position in the approximation are used. The correlation value is then mapped to the interval $[0 ; 1]$. Altogether, the correlation base weight at the position $\mathbf{x}$ is computed according to:

$$
G_{l}^{\text {corr }}(\mathbf{x})=\left(\frac{1}{2}\left(\frac{\operatorname{Cov}\left(A_{l-1}, B_{l-1}\right)}{\sqrt{\operatorname{Var}\left(A_{l-1}\right) \operatorname{Var}\left(B_{l-1}\right)}}+1\right)\right)^{p},
$$

with covariance

$$
\operatorname{Cov}(a, b)=\frac{1}{n} \sum_{\mathbf{x} \in \Omega_{\mathbf{x}}}(a(\mathbf{x})-\bar{a})(b(\mathbf{x})-\bar{b}),
$$

and variance

$$
\operatorname{Var}(a)=\frac{1}{n} \sum_{\mathbf{x} \in \Omega_{\mathbf{x}}}(a(\mathbf{x})-\bar{a})^{2},
$$

where $n$ defines the number of pixels in the neighborhood $\Omega_{\mathbf{x}}$ and $\bar{a}=\frac{1}{n} \sum_{\mathbf{x} \in \Omega_{\mathbf{x}}} a(\mathbf{x})$ defines the average value within $\Omega_{\mathbf{x}}$. The parameter $p$ in eq. (4) can be used for controlling the amount of noise reduction. With increasing $p$ coefficients with 
lower correlation are stronger suppressed. We use a simple square/cubic neighborhood defined as:

$$
\Omega_{\mathbf{x}}=\left\{\tilde{\mathbf{x}}|| \tilde{x}_{k}-x_{k} \mid \leq \frac{s}{2}, \forall k\right\} .
$$

with $k=\{1,2\}$ in $2 \mathrm{D}, k=\{1,2,3\}$ in $3 \mathrm{D}$ and parameter $s$ controlling the size of the neighborhood .

\section{Noise Estimation}

Additionally, for each orientation a local noise estimation is computed in order to assess the significance of each detail coefficient versus the noise level.

The difference $D=A-B=N_{A}-N_{B}$ between the two input datasets shows just noise. The question is how this difference can be used for estimating noise in the average of the two input datasets $M=\frac{1}{2}(A+B)$. If $A$ and $B$ are viewed as random variables, then

$$
L=g_{1} A+g_{2} B
$$

is their linear combination with weights $g_{1}, g_{2} \in \mathbb{R}$. The following holds for the variances [14]:

$$
\sigma_{L}^{2}=g_{1}^{2} \sigma_{A}^{2}+g_{2}^{2} \sigma_{B}^{2}+2 g_{1} g_{2} \operatorname{Cov}(A, B) .
$$

Since $A$ and $B$ include the same noise-free signal and zeromean noise, the covariance between $A$ and $B$ equals the covariance of the noise. Incorporating the two assumptions that noise is uncorrelated between the two input datasets (eq. 3) and that the amount of noise in both datasets is approximately the same (eq. 2), eq. 9 simplifies to:

$$
\sigma_{L}=\sqrt{g_{1}^{2}+g_{2}^{2}} \sigma_{A}
$$

Thus, the following relation between noise in the difference, each of the input datasets and the averaged input datasets can be derived:

$$
\sigma_{M}=\frac{\sigma_{A}}{\sqrt{2}}=\frac{\sigma_{B}}{\sqrt{2}}=\frac{\sigma_{D}}{2} .
$$

Due to the linearity of the wavelet transformation this can be used directly for estimating the local and orientation dependent standard deviation of noise in the different frequency bands of the wavelet decomposition. The differences between the detail coefficients are computed for all decomposition levels $l$ and all orientations d:

$$
W_{D, l}^{\mathrm{d}}=W_{A, l}^{\mathrm{d}}-W_{B, l}^{\mathrm{d}} .
$$

From these differences the corresponding standard deviations $\sigma_{l}^{\mathrm{d}}(\mathbf{x})$ are locally computed for all positions $\mathbf{x}$ according to:

$$
\sigma_{l}^{\mathrm{d}}(\mathbf{x})=\sqrt{\frac{1}{n} \sum_{\mathbf{x} \in \Omega_{\mathbf{x}}}\left(W_{D, l}^{\mathrm{d}}(\mathbf{x})\right)^{2}},
$$

From this estimation, significance-weights are computed for each detail coefficient:

$$
G_{l}^{\mathrm{sig}, \mathrm{d}}(\mathbf{x})= \begin{cases}1, & \left|W_{M, l}^{\mathrm{d}}(\mathbf{x})\right| \geq k \sigma_{l}^{\mathrm{d}}(\mathbf{x}), \\ e^{-\left(1-\left(\frac{W_{M, l}^{\mathrm{d}}(\mathbf{x})}{k \sigma_{l}^{\mathrm{d}}(\mathbf{x})}\right)^{2}\right)^{2}}, & \text { else }\end{cases}
$$

Averaged detail coefficients $W_{M, l}^{\mathrm{d}}(\mathbf{x})$ with absolute value above the local, noise dependent threshold $k \sigma_{l}^{\mathrm{d}}(\mathbf{x})$ are kept unchanged, values below are attenuated according to their difference to the threshold. The parameter $k$ controls the amount of noise suppression in relation to the noise power. The higher the $k$, the more noise is removed. With increasing parameter $r$ the significance weight tends more and more to an adaptive hard thresholding. In our experiments we used $r=10$.

\section{Weighting of Detail Coefficients}

Altogether, the averaged detail coefficients of $A$ and $B$ are weighted with the product of correlation based weight and significance-weight:

$$
W_{R, l}^{\mathrm{d}}(\mathbf{x})=W_{M, l}^{\mathrm{d}}(\mathbf{x}) \cdot G_{l}^{\mathrm{sig}, \mathrm{d}}(\mathbf{x}) \cdot G_{l}^{\mathrm{corr}}(\mathbf{x})
$$

for all directions $d$ and all decomposition levels $l=$ $1, \ldots, l_{\max }$. The approximation coefficients at the maximum decomposition level $l_{\max }$ are just averaged:

$$
R_{l}(\mathbf{x})=\frac{1}{2}\left(A_{l}(\mathbf{x})+B_{l}(\mathbf{x})\right) .
$$

The noise suppressed result $R$ is obtained by an inverse discrete wavelet transformation of the averaged and weighted coefficients.

\section{RESUlts}

In Fig. 2 an example slice, taken from a thoracic scan, is shown. The original slice (Fig. 2(a)) as well as the difference between the separate reconstructions (Fig. 2(b)) show directed noise due to high attenuation along the horizontal direction. An edge-detection is performed by correlation analysis between the approximation images, as can be seen in the weighting image at the first decomposition level in Fig. 2(e). In Fig. 2(f)-2(h) the combinations of correlation based weight and orientation dependent significance weights are shown for the horizontal, vertical and diagonal direction. This combination allows an adaptive, anisotropic denoising. The noise suppressed result image (3 levels of 2D-Haar-DWT, denoised with $p=1.0, k=1.5$ ) is shown in Fig. 2(c), together with the difference to the original in Fig. 2(d).

In Fig. 3 results are presented for a thin reconstructed slice $(0.8 \mathrm{~mm})$ taken from a CTA of a liver (see also difference images). The original noisy slice is shown in Fig. 3(a). Fig. 3(b) shows the result of a 2D nonlinear diffusion (Perona and Malik PDE using a Tukey edge stopping-function [15] and a multigrid-solver) in order to illustrate the difficulties of denoising CT data with standard methods. In the center, where noise is stronger, nearly no noise has been removed, while the outer regions were already blurred. In Fig. 3(c) the denoising result of the proposed method in $2 \mathrm{D}$ is presented ( $p=1.0, k=1.5$ ). Noticeably, the method adapted itself to the noise power in the image and removed noise more uniformly. In average, we achieved a reduction of pixel noise (standard deviation of noise in homogeneous region) of approximately $45 \%$ in $2 \mathrm{D}$. Nevertheless, the overall image appearance is not very "natural" with respect to the residual noise power 


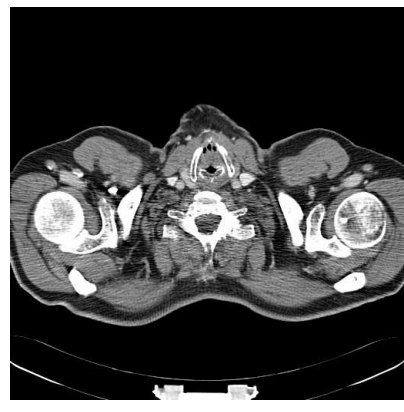

(a) Original $((A+B) / 2)$

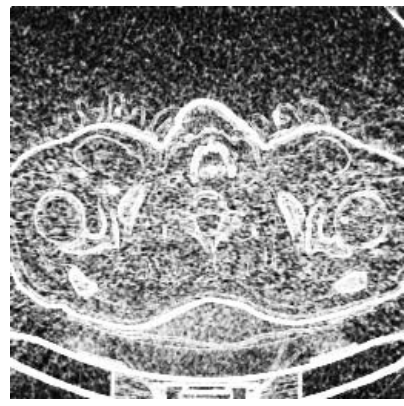

(e) Correlation weight

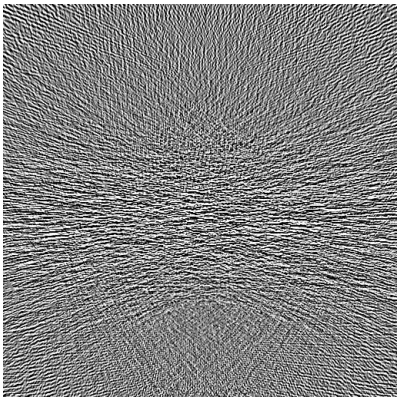

(b) $(A-B) / 2$

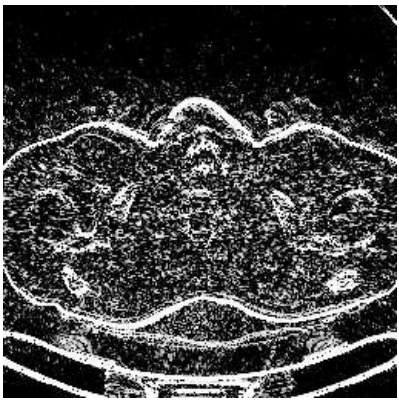

(f) Horizontal weight

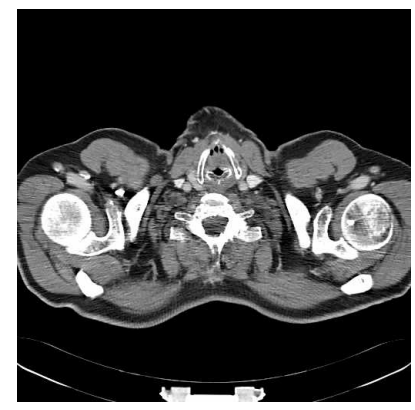

(c) Denoised

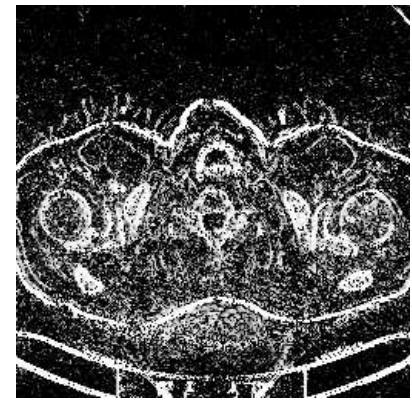

(g) Vertical weight

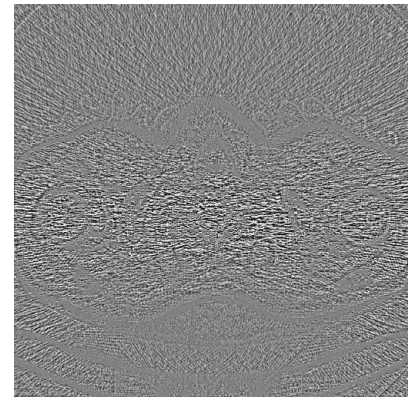

(d) Denoised-Original

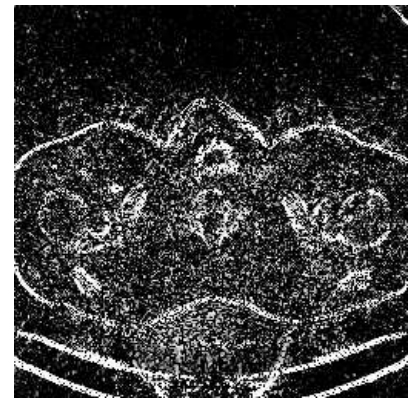

(h) Diagonal weight

Fig. 2. Denoising results for a thoracic slice, displayed with $c=50$ and $w=400$. Difference images are displayed with $c=0$ and $w=100$. The corresponding correlation based weight and the combinations with orientation dependent significance weights are shown for the first decomposition level $(0$ corresponds to black, 1 corresponds to white).

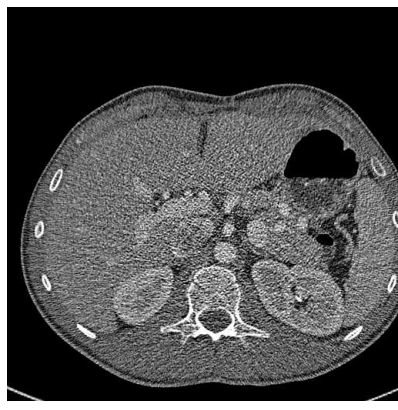

(a) Original $((A+B) / 2)$

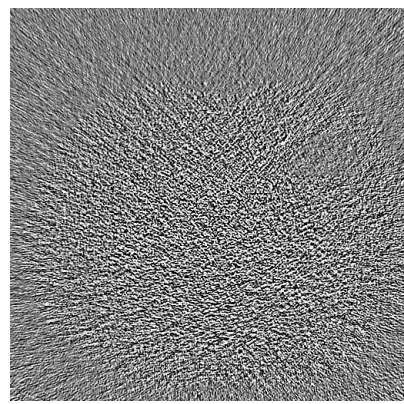

(e) $(A-B) / 2$

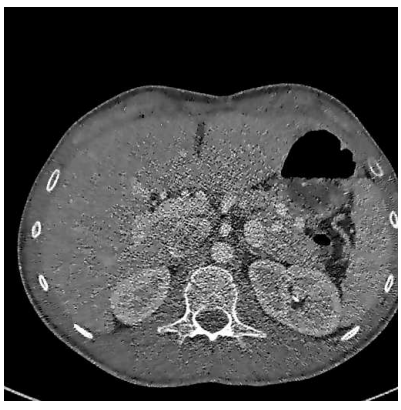

(b) 2D Diffusion

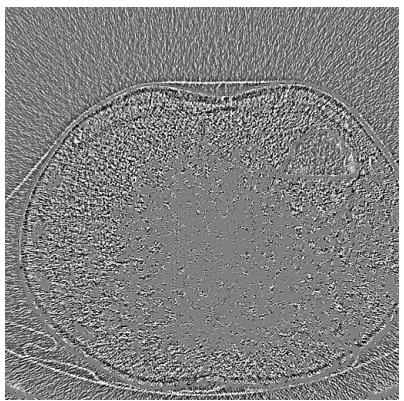

(f) Diffusion-Original

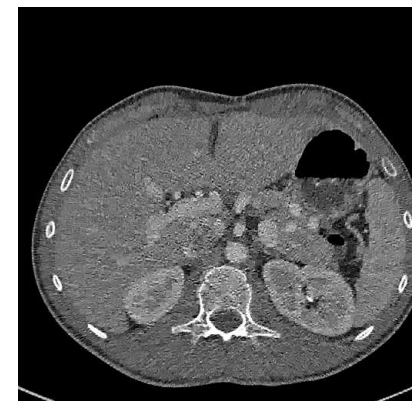

(c) 2D Denoised

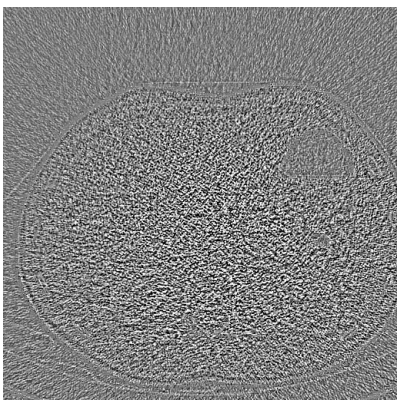

(g) 2D Denoised-Original

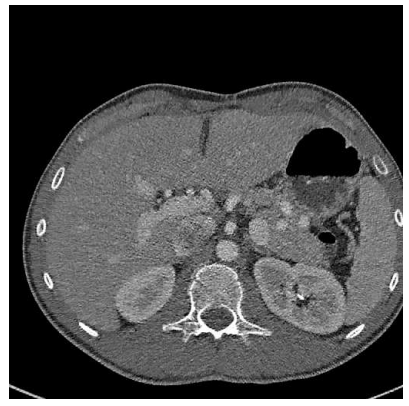

(d) 3D Denoised

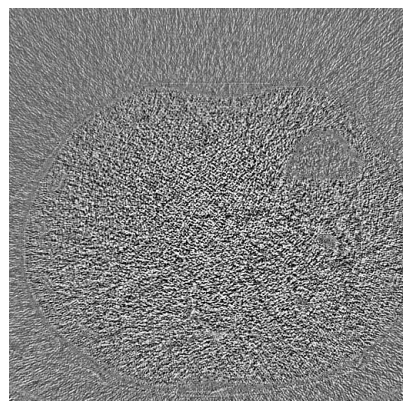

(h) 3D Denoised-Original

Fig. 3. Denoising results of different approaches for a CTA of a liver, displayed with $c=200$ and $w=700$. The corresponding difference images are displayed with $c=0$ and $w=200$. 


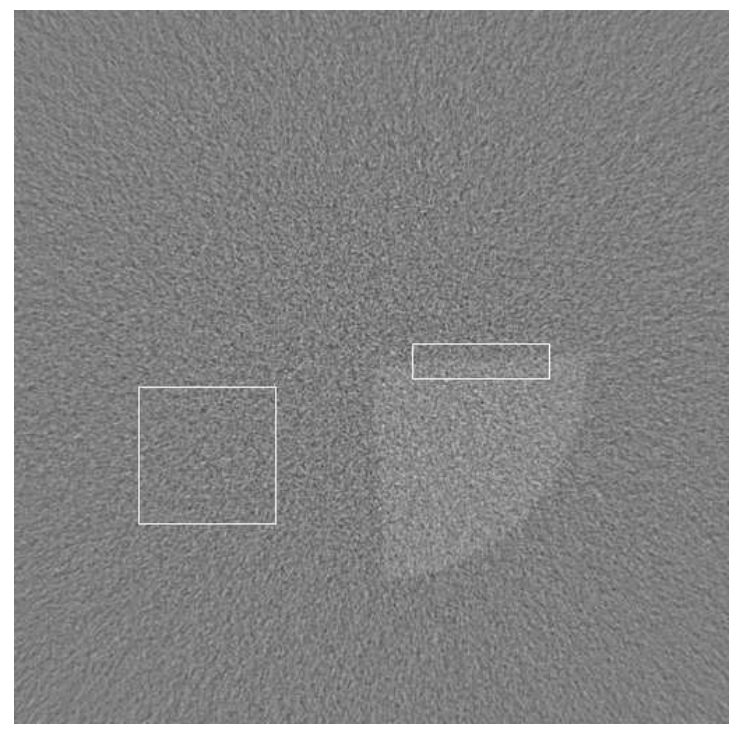

Fig. 4. Noisy example slice with $10 \mathrm{HU}$ contrast at the edge $(c=0, w=$ 200 ), together with regions used for quantitative evaluation of noise and resolution.

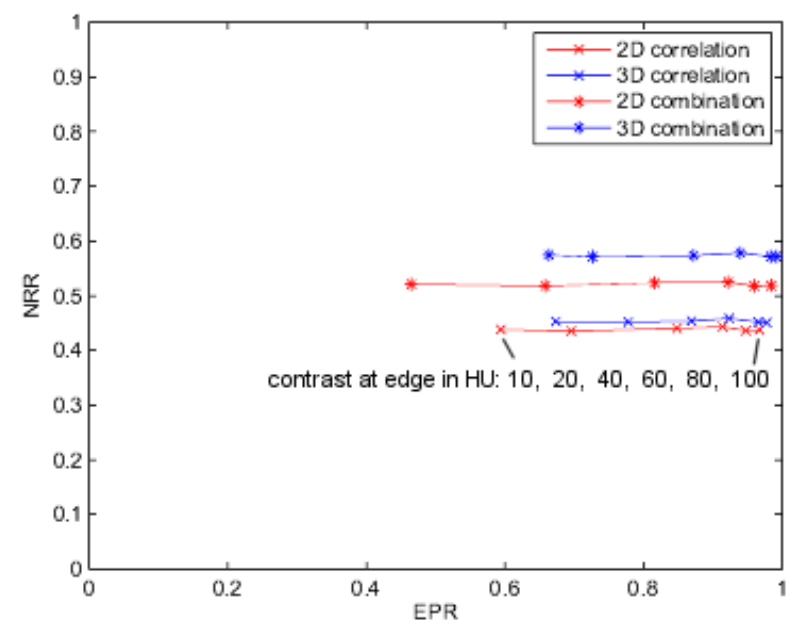

Fig. 5. Comparison of noise reduction rate and edge-preservation of denoising with correlation based weighting and the combination of correlation and significance weights in $2 \mathrm{D}$ and $3 \mathrm{D}$.

spectrum. The reason for this is that we used only a small neighborhood of $5 \times 5$ pixels for correlation computation. Therefore, the correlation analysis is not very reliable and some false correlations lead to noise remaining in the image. In comparison, the denoising in 3D with $5 \times 5 \times 5$ neighborhoods and same parameter settings, shown in Fig. 3(d), is more effective. Noise is removed very well (up to 60\%) in homogeneous areas and also close to edges. Even at lower contrasts, edges are still preserved. For example, the contrasted vessels in the liver are better visible in the noise suppressed image in comparison to the original.

In addition to the visual inspection of real clinical data, quantitative tests were performed evaluating noise reduction and edge preservation based on phantom data. For generating the simulated CT-scans the DRASIM software package, provided by Karl Stierstorfer [16], was used. The considered analytical phantoms consisted of a water cylinder (radius $=$ $15 \mathrm{~cm}$ ) with an inlaid quartered cylinder (radius $=6 \mathrm{~cm}$ ) of defined contrast. In order to test the preservation of edges at different contrast-to-noise levels the density of the object was varied leading to edge-contrasts between 10 to 100 Hounsfield units (HU). An example slice with a contrast of $10 \mathrm{HU}$ is shown in Fig. 4. The average standard-deviation of noise in the homogeneous region of the water phantom was kept constant at approximately $\sigma^{\text {original }}=10.6 \mathrm{HU}$. All tests $(2 \mathrm{D}$ and $3 \mathrm{D}$ using 3 levels of a Haar-DWT with $p=1.0, k=1.5$ ) were performed on 16 neighboring slices $(512 \times 512 \times 16)$. For evaluating the noise reduction rate we measured the standard deviation of the pixel values within a homogeneous region of the water phantom of $100 \times 100$ pixels in all 16 slices, build the average and compared it with the mean noise in the original. Accordingly, the noise reduction rate (NRR) is defined as:

$$
\mathrm{NRR}=1-\frac{\sigma^{\text {denoised }}}{\sigma^{\text {original }}} \text {. }
$$

In addition to noisy phantoms with Poisson distributed noise, ideal CT-scans were simulated leading to noise-free ground-truth data. In CT a standard measurement for resolution is the modulation transfer function (MTF) (see e.g. [12]), indicating how many line pairs per $\mathrm{cm}(\mathrm{lp} / \mathrm{cm})$ can be distinguished. It is possible to determine the local MTF directly from the edge in an image. For this purpose, we manually selected a fixed region of $20 \times 125$ pixels around an edge (with a slope of approx. 4 degrees). The slight tilt of the edge allows a higher sampling of the edge profile, which is additionally averaged along the edge. The derivation of the edge profile leads to the line-spread function (LSF). The Fourier transformation of the LSF results in the MTF, which is additionally normalized so that $\operatorname{MTF}(0)=1$. Reliable measurements of the MTF from this edge technique can only be achieved if the contrast of the edge is much higher than the pixel noise [17]. Due to the linearity of the wavelet transformation the computed weights gained from the correlation analysis and noise estimation can directly be applied to the wavelet coefficients of the ideal noise-free data. The computation of the inverse wavelet transformation of these weighted coefficients makes the influence of the denoising process to the ideal signal directly visible. The local MTF can then be computed at the edge in the processed noise-free data. For comparing the edgepreservation of the different methods at different contrasts we used the $\rho_{50}$ value. This is the spatial frequency for which the MTF reaches a value of 0.5 . This value is set into relation to the resolution in the original noise-free signal. We define the edge-preservation rate (EPR) as:

$$
\mathrm{EPR}=\frac{\rho_{50}^{\text {denoised }}}{\rho_{50}^{\text {original }}}
$$

where $\rho_{50}^{\text {original }}$ is the resolution in the original and $\rho_{50}^{\text {denoised }}$ the resolution at the denoised data.

In Fig. 5 the NRR is plotted against the EPR. The correlation based weighting (correlation) is compared to the combination of correlation based weighting and orientation dependent significance-weights (combination). Furthermore, a 
comparison between the $2 \mathrm{D}$ and $3 \mathrm{D}$ results is shown. The NRR and EPR is in all cases plotted for the different datasets using different contrasts at the edge. It can be seen, that in all cases the edge-preservation depends on the contrast-to-noise level of the input data. The higher the contrast of an edge, the better the edge can be detected and preserved. It can be observed that for higher contrasts at the edge the proposed combination of correlation based weight and significance-weight leads to higher noise reduction and better edge preservation. For lower contrasts, however, a higher noise reduction can only be achieved with a slightly stronger smoothing at the edge. The application of the denoising method in $3 \mathrm{D}$ in comparison to 2D generally leads to higher noise-suppression in combination with better edge-preservation. Especially for the 3D case, nearly no difference in edge-preservation can be observed between the correlation based weight and the combination with significance-weights. The average noise reduction rate improved from approximately $44 \%$ (2D correlation) to $58 \%$ (3D combined).

\section{CONCLUSIONS}

We presented a new 3D wavelet based method for structurepreserving noise reduction in reconstructed CT volumes. The method adapts itself to the locally varying noise power. Further, the combination of correlation analysis and orientation dependent noise estimation allows for an anisotropic denoising. The anisotropic behavior is especially beneficial for datasets with directed noise. The extension of the method to $3 \mathrm{D}$ results in higher noise reduction, up to $60 \%$, and even better edge-preservation.

\section{ACKNOWLEDGMENT}

The authors gratefully thank Karl Stierstorfer, Holger Kunze and Elli Angelopoulou for helpful suggestions and discussions. Further, the authors acknowledge the financial support by Siemens Medical Solutions, the International Max-Plack Research School on Optics and Imaging, and the MIC 2007 for awarding the MIC trainee grant.

\section{REFERENCES}

[1] W. A. Kalender, Computed Tomography. Erlangen: Publicis Corporate Publishing, 2000.

[2] J. Fessler, et al., "Grouped-coordinate ascent algorithms for penalizedlikelihoodtransmission image reconstruction," IEEE Trans Med Imaging, vol. 16, no. 2, pp. 166-175, April 1997.

[3] $\mathrm{T}$. Li, et al., "Nonlinear sinogram smoothing for low-dose X-ray CT," IEEE Transactions on Nuclear Science, vol. 51, no. 5, pp. 2505-2512, October 2004.

[4] P. La Rivire, et al., "Penalized-likelihood sinogram restoration for computed tomography." IEEE Trans Med Imaging, vol. 25, no. 8, pp. 1022-1036, August 2006.

[5] J. Hsieh, "Adaptive streak artifact reduction in computed tomography resulting from excessive x-ray photon noise," Medical Physics, vol. 25, no. 11 , pp. 2139-2147, November 1998.

[6] M. Kachelrieß, et al., "Generalized multi-dimensional adaptive filtering for conventional and spiral single-slice, multi-slice, and cone-beam CT," Medical Physics, vol. 28, no. 4, pp. 475-490, April 2001.

[7] O. Demirkaya, "Reduction of noise and image artifacts in computed tomography by nonlinear filtration of projection images," in Medical Imaging 2001: Image Processing. Proceedings of the SPIE., M. Sonka and K. M. Hanson, Eds., vol. 4322, July 2001, pp. 917-923.
[8] O. Tischenko, et al., "An artifact-free structure-saving noise reduction using the correlation between two images for threshold determination in the wavelet domain," in Medical Imaging 2005: Image Processing. Proceedings of the SPIE., J. M. Fitzpatrick and J. M. Reinhardt, Eds., vol. 5747, April 2005, pp. 1066-1075.

[9] A. Borsdorf, et al., "Wavelet based Noise Reduction by Identification of Correlations ," in Pattern Recognition (DAGM 2006), Lecture Notes in Computer Science , K. Franke, et al., Eds., vol. 4174. Berlin: Springer, 2006, pp. 21-30.

[10] A. Borsdorf, et al., "Separate CT-Reconstruction for Orientation and Position Adaptive Wavelet Denoising ," in Bildverarbeitung für die Medizin 2007 , A. Horsch, et al., Eds. Berlin: Springer, 2007, pp. 232-236.

[11] A. Kak and M. Slanely, Principles of Computerized Tomographic Imaging. Society for Industrial and Applied Mathematics, July 2001, http://www.slaney.org/pct/pct-toc.html.

[12] T. Buzug, Einführung in die Computertomographie. Berlin Heidelberg: Springer-Verlag, 2004.

[13] E. W. Weisstein, "Correlation coefficient," 2006, from MathWorld - A Wolfram Web Resource, http://mathworld.wolfram.com/CorrelationCoefficient.html.

[14] E. W. Weisstein, "Variance," February 2006, from MathWorld - A Wolfram Web Resource, http://mathworld.wolfram.com/Variance.html.

[15] M. Black, et al., "Robust anisotropic diffusion," IEEE Trans. Med. Imag., vol. 7, no. 3, pp. 421-432, March 1998.

[16] K. Stierstorfer, et al., "Segmented multiple plane reconstruction: A novel approximate reconstruction scheme for multi-slice spiral CT," Physics in Medicine and Biology, vol. 47, no. 4, pp. 2571-2581, July 2002.

[17] I. A. Cunningham and B. K. Reid, "Signal and noise in modulation transfer function determinations using the slit, wire, and edge techniques," Medical Physics, vol. 19, no. 4, pp. 1037-1044, July 1992. 